\title{
Rasgos de personalidad vinculados a la percepción del rendimiento académico de estudiantes universitarios de música
}

\section{Personality traits linked to the perception of the academic performance of university music students}

AGUIRRE-ASCACIO, Miguel Moctezumat*, HERNÁNDEZ-CUETO, Jaquelina Lizet, YAÑEZFLORES, Sara Margarita y SALINAS-AGUIRRE, María del Consuelo

Universidad Autónoma de Coahuila

ID $1^{\text {er }}$ Autor: Miguel Moctezuma, Aguirre Ascacio / ORC ID: 0000-0001-6388-0402, Researcher ID Thomson: X-33502019

ID $1^{\text {er }}$ Coautor: Jaquelina Lizet, Hernández Cueto / ORC ID: 0000-0002- 3728- 7434, Researcher ID Thomson: S-85882018, CVU CONACYT ID: 322702

ID $2^{\text {do }}$ Coautor: Sara Margarita, Yañez Flores / ORC ID: 0000-0002-4750-4244, Researcher ID Thomson: S-9231-2018, CVU CONACYT ID: 352125

ID $3^{\text {er }}$ Coautor: María Del Consuelo, Salinas Aguirre / ORC ID: 0000-0002-6542-1813, Researcher ID Thomson: S-92442018, CVU CONACYT ID: 615335

DOI: $10.35429 / J T M S .2019 .17 .5 .1 .8$

Recibido 04 de Septiembre 2019; Aceptado 30 Diciembre, 2019

\section{Resumen}

La presente investigación aborda la personalidad partir del trabajo desarrollado por el psicólogo alemán Hans Jurgen Eysenck y su cuestionario de personalidad y el rendimiento académico se genera a partir de ideas desarrolladas por Vargas en donde enfoques individuales, familiares e institucionales son tomados en cuenta. El objetivo es conocer la relación subyacente entre la personalidad y la percepción del rendimiento académico en estudiantes de música a nivel licenciatura. Por medio de un diseño cuantitativo, no experimental, transversal se caracteriza a la población y se correlaciona la información recabada. La metodología es una correlación Momento Producto de Pearson, entre los tópicos ya descritos. La contribución es ofrecer un estudio objetivo sobre lo que este colectivo en particular considera con respecto a su rendimiento académico y la manera en que incide en ello la personalidad de los alumnos. Con lo cual se abre un espectro diferente en cuanto a la emotividad y sensibilidad de los comportamientos de los sujetos y el contexto educativo.

Percepción, Personalidad, Rendimiento Académico

\begin{abstract}
This research addresses personality from the work developed by The German psychologist Hans Jurgen Eysenck and his personality questionnaire and academic performance is generated from ideas developed by Vargas where they approach individual, family and institutional are taken into account. The goal is to know the underlying relationship between personality and perception of academic performance in music students at the bachelor's level. Quantitative, non-experimental, transverse design characterizes the population and correlates the information collected. The methodology is a correlation Moment Product of Pearson, among the topics already described. The contribution is to provide an objective study on what this particular group considers with regard to its academic performance and how it affects the personality of the students. This opens up a different spectrum in terms of the emotion and sensitivity of subject behaviors and educational context.
\end{abstract}

Perception, Personality, Academic Performance

Citación: AGUIRRE-ASCACIO, Miguel Moctezuma, HERNÁNDEZ-CUETO, Jaquelina Lizet, YAÑEZ-FLORES, Sara Margarita y SALINAS-AGUIRRE, María del Consuelo. Revista Transdisciplinaria de Estudios Migratorios. 2019, 5-17: $1-8$

\footnotetext{
* Correspondencia al Autor (correo electrónico: agasmi985@ hotmail.com)

$\dagger$ Investigador contribuyendo como primer Autor.
} 


\section{Introducción}

A lo largo del tiempo los estudios encaminados a comprender los aspectos relacionados al rendimiento académico han partido de principios aptitudinales y cognitivos, de acuerdo a Catell (1965), constructos psicológicos como la inteligencia y la aptitud pueden llegar a explicar el 25 por ciento de la varianza total del rendimiento académico. Sin embargo, en años recientes diversas investigaciones señalan la escasa capacidad de predicción que ofrecen factores exclusivamente cognitivos o aptitudinales sobre el rendimiento académico a largo plazo y el éxito laboral fuera de entornos educativos.

Cada vez más investigaciones coinciden en señalar la relación que tiene el estudio de la personalidad sobre el rendimiento académico destacando la importancia de realizar aproximaciones desde enfoques grupales ante la imposibilidad de hacer inferencias y generalizaciones válidas derivadas del estudio de casos particulares.

En respuesta a lo anterior, el presente estudio tiene como objetivo principal conocer la relación subyacente entre la personalidad y el rendimiento académico en estudiantes de música a nivel licenciatura, lo anterior debido a que una de las problemáticas principales en distintas escuelas de música del país a nivel superior es precisamente el bajo rendimiento académico.

Se estructuran antecedentes a partir de la revisión de investigaciones relacionadas al tema en donde diversos autores exponen sus principales hallazgos; si bien dichos hallazgos se derivan de estudios en poblaciones con diferente perfil profesional es conveniente mencionar que de acuerdo a Eysenck (1987), las actitudes y comportamientos de las personas son invariables en situaciones temporales y espaciales, por lo que los hallazgos encontrados tienen la misma validez. Posteriormente, se hace una descripción detallada sobre los conceptos principales y las teorías desarrolladas a partir de los constructos que guían la temática del presente estudio con la finalidad de establecer límites conceptuales a los que se ajusta este artículo. A partir de este punto que se toma la decisión de desarrollar el estudio con un diseño cuantitativo, no experimental y transversal con alcance correlacional.
Dentro de los resultados se trabaja el nivel correlacional encontrándose numerosas relaciones con significancia estadística que validan los resultados encontrados, mismos que son la evidencia empírica que soportan las conclusiones que se exhiben.

\section{Metodología a desarrollar}

El presente estudio tiene un enfoque cuantitativo, ya que se afina y estructura la idea de investigación a partir de la articulación coherente e integrada de elementos que pueden ser objeto de mediciones empíricas a partir de la recolección de datos que prueben o anulen la validez de las hipótesis planteadas con base en la medición numérica y el análisis estadístico. Se elabora un análisis de frecuencia y porcentaje para la descripción de la muestra y un análisis correlacional Momento Producto de Pearson.

Tiene un diseño no experimental y transversal ya que, de acuerdo a Hernández, Fernández y Baptista (2008, p.209) no se pretende la manipulación deliberada de ninguna variable, es decir, solo se observan situaciones ya existentes (personalidad y percepción del rendimiento académico) de manera que no es posible manipularlas, ni tener control directo o influir sobre ellas porque ya sucedieron al igual que sus efectos; a su vez es transversal; ya que la recopilación de los datos ocurrió en un solo punto del tiempo y a partir de ahí su propósito es la descripción y análisis de incidencias e interrelaciones en un momento dado.

Para la obtención de datos se diseñó un instrumento tipo encuesta consistente en treinta y dos variables simples de medición numérica, decimal de razón; distribuidas en cinco dimensiones para el caso del eje de personalidad. Con respecto al eje de rendimiento académico, éste fue medido a través de doce variables simples, con escala numérica decimal de razón. Para conocer las características de la población se preguntaron seis datos del respondiente, correspondientes a sexo, edad, semestre, especialidad, promedio general logrado en educación media superior y si vive con sus padres. Variables de tipo nominal, a excepción del promedio que finalmente no fue considerada en el desarrollo estadístico del trabajo debido a no tener $n$ suficiente. 


\section{Resultados}

\section{Frecuencias y porcentajes}

A continuación se realiza una descripción de la población que conforma el presente estudio en relación al sexo, edad, semestre y situación parental (vive con padres-no vive con padres) expresados en frecuencias y porcentajes.

Se observa la población de estudio se conforma por 70 hombres y 35 mujeres, es decir, una proporción de $66.7 \%$ de hombres por un 33.3 de mujeres. Las edades de los encuestados oscilan entre los 15 y los 31 años, teniendo mayor incidencia en el rango de los 17 a los 20 años.

La concentración del grueso de la muestra se encuentra en el segundo semestre de carrera. La cantidad de estudiantes que viven con sus padres es de 71 (67.7\%) mientras que los que no viven con sus padres suman 33 estudiantes (31.4\%), tan solo 1 estudiante no contestó.

\section{Correlación}

Se realizó el análisis de correlación producto momento de Pearson con un $95 \%$ de confianza a las variables que integran las dimensiones Extroversión-Introversión y Percepción del rendimiento obteniéndose los siguientes resultados:

\section{Correlación de Pearson de las dimensiones Extraversión-Introversión y Percepción del Rendimiento Académico}

Se observa que a mayor irresponsabilidad menor tiempo de preparación y menor calidad de estudio previo a los exámenes, a la vez que hay menor comodidad en las evaluaciones. Por otra parte se observa que a mayor irresponsabilidad mayor es el nivel de comparación que tienen los estudiantes con sus compañeros y mayor es la percepción de éxito fortuito.

Además se observa que a mayor nivel de sociabilidad mayor es la percepción de que las calificaciones no son confiables y mayor es también la percepción de éxito fortuito. Por último se observa que a mayor tendencia a la impulsividad mayor es la percepción de éxito fortuito y mayor es también la inseguridad en los estudiantes.
Se infiere que estudiantes con rasgos de personalidad inclinados hacia conductas irresponsables como la impuntualidad, la improvisación y la falta de rigurosidad en las actividades escolares tienden a considerar que se preparan mal y con poco tiempo de anticipación antes de presentar sus exámenes, mismos en los que consideran sentirse incomodos; además de pensar que sus compañeros obtendrán mejores resultados que ellos en cualquier evaluación y en caso de obtener un buen resultado lo atribuyen a su buena suerte.

Por otro lado se observa que los estudiantes con rasgos de personalidad inclinados hacia conductas sociables como salir con frecuencia, relacionarse y sentirse a gusto en la compañía de muchas personas consideran que un buen rendimiento académico se debe más a su buena suerte que a su propia habilidad, al mismo tiempo ellos manifiestan que sus calificaciones no reflejan su verdadero nivel de conocimiento. Estudiantes con rasgos inclinados hacia la impulsividad, reflejada en conductas como comprar por impulso, hacer las cosas por la emoción del momento o meterse en problemas por hacer las cosas sin pensar, consideran que su buen rendimiento académico se debe más a su buena suerte que a las habilidades o conocimientos que poseen y tienden a pensar que al momento de realizar cualquier prueba su rendimiento no será el esperado.

\begin{tabular}{|l|l|l|}
\hline Variables Correlacionadas & \multicolumn{1}{c|}{ r } & Sig. \\
\hline Irrespo/Compa & 0.215 & 0.028 \\
\hline Irrespo/Pr Previa & -0.389 & 0 \\
\hline Irrespo/B Prep & -0.294 & 0.002 \\
\hline Irrespo/Com eva & -0.304 & 0.002 \\
\hline Irrespo/Éx Fort & 0.204 & 0.037 \\
\hline Socia/Éx fort & 0.238 & 0.014 \\
\hline Socia/Cal no con & 0.206 & 0.036 \\
\hline Impul/Éx Fort & 0.208 & 0.034 \\
\hline Impul/Ins & 0.238 & 0.014 \\
\hline
\end{tabular}

Tabla 1 Extraversión y R. Académico Fuente: Elaboración Propia

\section{Correlación de Pearson de las dimensiones Estabilidad-Inestabilidad emocional y Percepción del Rendimiento Académico}

Se observa una correlación positiva entre culpa y las variables comparación, nerviosismo, inseguridad, tristeza y éxito fortuito mientras que el rasgo culpa dada la carga negativa del coeficiente de correlación; no se asocia con las variables preparación previa, buena preparación y comodidad en la evaluación. 
También se observa una correlación positiva entre el rasgo hipocondría y la variable comodidad en evaluación mientras que negativa entre hipocondría y éxito fortuito.

Además se observa una correlación de carga positiva entre el rasgo ansiedad y las variables nerviosismo e inseguridad mientras que las variables buena preparación y comodidad en la evaluación tienen una correlación de carga negativa con el mismo rasgo.

Por otro lado se observa una correlación positiva entre el rasgo infelicidad y las variables comparación, tristeza y éxito fortuito mientras que se observa una correlación con carga negativa entre las variables preparación previa y comodidad en la evaluación con el rasgo anteriormente referido.

Por último el rasgo baja autoestima tiene una correlación positiva con las variables comparación, nerviosismo e inseguridad.

Se infiere que estudiantes con rasgos inclinados a la culpa como aquellos que manifiestan sentir la desaprobación de los demás o sentir que los placeres de hoy se tendrán que pagar en el futuro tienden a manifestar nervios además de manifestar inseguridad de que sus conocimientos sean suficientes, que su rendimiento no será el esperado en las pruebas y que los compañeros obtendrán mejores resultados; estos estudiantes manifiestan sentimientos de tristeza cuando obtienen malos resultados y en caso de que estos sean positivos le atribuyen mucha importancia a la buena suerte. Por otro lado se observa que estudiantes con inclinación a la culpa consideran dedicar poco tiempo y poca calidad de estudio previo a un exámen además de sentirse incomodos en los procesos de evaluación.

Los estudiantes que se alteran con facilidad, les cuesta olvidar un error propio o muestran demasiada preocupación por las cuestiones monetarias consideran sentirse inseguros de sus conocimientos y nerviosos previo a cualquier examen, estos estudiantes consideran no prepararse con la suficiente calidad y se sienten muy incomodos en los procesos de evaluación.
Cabe mencionar que estudiantes inclinados hacia rasgos de infelicidad como sentirse deprimidos constantemente, sentir placer con las desgracias de los demás o mostrar pesimismo de cara al futuro consideran a menudo que sus compañeros obtendrán mejores resultados que ellos en las evaluaciones, tienen sentimientos de tristeza al obtener malas calificaciones aún sin haber estudiado y en caso de que los resultados sean positivos suelen atribuirle mucha importancia a la buena suerte, estos estudiantes no estudian con anticipación a sus exámenes y sienten incomodidad en los procesos de evaluación. Estudiantes con autoestima baja tienden a manifestar inseguridad de sus propios conocimientos y de tener sentimientos de inferioridad respecto a las calificaciones que obtendrán sus compañeros en las pruebas además de manifestar nerviosismo en los exámenes.

\begin{tabular}{|l|r|r|}
\hline Variables correlacionadas & \multicolumn{1}{c|}{ r } & Sign. \\
\hline Cul/Compa & $.331^{* *}$ & 0.001 \\
\hline Cul/Nerv & $.302^{* *}$ & 0.002 \\
\hline Cul/Ins & $.285^{* *}$ & 0.003 \\
\hline Cul/Tris & $.281^{* *}$ & 0.004 \\
\hline Cul/Éx fort & $.235^{*}$ & 0.016 \\
\hline Cul/Pr previa & $-.232^{*}$ & 0.017 \\
\hline Cul/B prep & $-.245^{*}$ & 0.012 \\
\hline Cul/Com eva & $-.253^{* *}$ & 0.009 \\
\hline Hip/Com eva & $.272^{* *}$ & 0.005 \\
\hline Hip/Éx fort & $-.270^{* *}$ & 0.005 \\
\hline Ans/Nerv & $.288^{* *}$ & 0.003 \\
\hline Ans/Inse & $.219^{*}$ & 0.025 \\
\hline Ans/B prep & $-.276^{* *}$ & 0.004 \\
\hline Ans/Com eva & $-.268^{* *}$ & 0.006 \\
\hline Inf/Compa & $.240^{*}$ & 0.014 \\
\hline Inf/Tris & $.291^{* *}$ & 0.003 \\
\hline Inf/Éx fort & $.332^{* *}$ & 0.001 \\
\hline Inf/Pr previa & $-.223^{*}$ & 0.022 \\
\hline Inf/Com eva & $-.229^{*}$ & 0.019 \\
\hline Baja aut/Compa & $.238^{*}$ & 0.015 \\
\hline Baja aut/Nerv & $.243^{*}$ & 0.012 \\
\hline Baja aut/Inse & $.361^{* *}$ & 0 \\
\hline
\end{tabular}

Tabla 2 Estabilidad y R. Académico

Fuente: Elaboración Propia

\section{Correlación de Pearson de la dimensión Psicotismo y Percepción del rendimiento Académico}

Se observa que la variable ambición tiene correlaciones negativas con las variables comparación y éxito fortuito, esta misma variable tiene correlaciones de tipo positivo con las variables preparación previa, buena preparación y comodidad en la evaluación. Por su parte se observa que la variable asertividad tiene correlaciones negativas con las variables comparación, inseguridad y éxito fortuito. 
Por último se observa una correlación negativa entre las variables agresividad y buena preparación y una correlación positiva entre las variables masculinidad y calificaciones no confiables. Se infiere que estudiantes con rasgos inclinados hacia la ambición, reflejada en conductas como establecer aspiraciones altas, perseguir los objetivo trazados con alta determinación sin dejar de trabajar un solo día o manifestar la necesidad de causar algún impacto en el mundo, tienden a estudiar con anticipación y buena calidad para presentar sus exámenes, mismos en los que consideran sentirse a gusto al momento de presentarlos, cabe señalar que estudiantes con un rasgo de personalidad ambiciosa no le atribuyen importancia al factor suerte en la obtención de buenos resultados académicos además de no creer que sus compañeros obtendrán mejores resultados que ellos en cualquier evaluación a la que sean sometidos. Respecto a aquellos estudiantes con rasgos de asertividad (reflejada en conductas como el hacer las cosas a su manera sin importar lo que piensen los demás, establecer límites en el trato con otros o no sentirse intimidado ante figuras de autoridad), manifiestan seguridad de que su rendimiento será optimo, además de no considerar que los compañeros obtendrán mejores resultados que ellos en las evaluaciones, cabe señalar que este tipo de estudiantes le atribuyen poco peso a la suerte en el logro de buenos resultados académicos.

Por último, es de llamar la atención la relación positiva que hay entre los rasgos de personalidad tendientes hacia la masculinidad (manifestada en conductas como la preferencia a participar en actividades toscas, el gusto por escenas de sexo o violencia en las películas o la preferencia por sintonizar juegos competitivos en tv como el box o el futbol) y la percepción de que las calificaciones obtenidas no reflejan en realidad los conocimientos que poseen los estudiantes.

\begin{tabular}{|l|r|r|}
\hline Variables correlacionadas & r & Sign. \\
\hline Masc/Cal no conf & $.208^{*}$ & 0.034 \\
\hline Amb/Compa & $-.219^{*}$ & 0.024 \\
\hline Amb/Éx fort & $-.333^{* *}$ & 0.001 \\
\hline Amb/Pr previa & $.234^{*}$ & 0.016 \\
\hline Amb/B prep & $.209^{*}$ & 0.032 \\
\hline Amb/Com en eva & $.212^{*}$ & 0.03 \\
\hline Aser/Compa & $-.260^{* *}$ & 0.007 \\
\hline Aser/Ins & $-.250^{* *}$ & 0.01 \\
\hline Aser/Éx fort & $-.236^{*}$ & 0.015 \\
\hline Agres/B prep & $-.232^{*}$ & 0.017 \\
\hline
\end{tabular}

Tabla 3 Neuroticismo y R. Académico

Fuente: Elaboración Propia

\section{Correlación de Pearson de las dimensiones Sexualidad y Percepción del rendimiento Académico}

Se observa una correlación negativa entre la variable tolerancia sexual y las variables desconfianza, comparación e inseguridad. Por otra parte la variable satisfacción sexual tiene correlaciones de sentido negativo con las variables comparación, nerviosismo, inseguridad, tristeza y éxito fortuito.

Por último la variable libido sexual tiene correlaciones de sentido negativo con las variables inseguridad, preparación previa y buena preparación.

Se infiere que los estudiantes con alta tolerancia sexual tienden a sentir mayor confianza sobre su rendimiento en las pruebas a los que son sometidos y tienen mayor confianza en que sus conocimientos sean suficientes para contestar adecuadamente, estos estudiantes no se comparan con los demás compañeros y manifiestan confianza en que obtendrán iguales o mejores resultados que sus compañeros en cualquier evaluación.

Aquellos estudiantes satisfechos con su vida sexual manifiestan tener mucha confianza sobre el rendimiento en cualquier prueba a la que son sometidos y confían en que sus conocimientos sean suficientes para contestar adecuadamente, además no se sienten inferiores a sus compañeros respecto a los resultados esperados en las evaluaciones, estos estudiantes consideran que sus resultados académicos son producto de su propia habilidad y conocimiento y le atribuyen poca importancia al factor suerte.

Por último, es de llamar la atención que aquellos estudiantes que manifiestan bajo nivel de deseo sexual tienden a ser inseguros respecto a que los conocimientos que poseen al momento de presentar los exámenes a los que son sometidos, a la vez que consideran prepararse de buena manera y con suficiente tiempo de anticipación para presentar sus exámenes. 


\begin{tabular}{|c|c|c|}
\hline Variables correlacionadas & $\mathbf{r}$ & Sign. \\
\hline Tol sex/Desc & $-.280^{* *}$ & 0.004 \\
\hline Tol sex & $-.267^{* *}$ & 0.006 \\
\hline Tol sex/Ins & $-.217^{*}$ & 0.026 \\
\hline Sat sex/Compa & $-.262^{* *}$ & 0.007 \\
\hline Sat sex/Nerv & $-.218^{*}$ & 0.025 \\
\hline Sat sex/Ins & $-.271^{* *}$ & 0.005 \\
\hline Sat sex/Tris & $-.211^{*}$ & 0.031 \\
\hline Sat sex/Éx fort & $-.225^{*}$ & 0.021 \\
\hline Líbido/Inse & $-.276^{* *}$ & 0.004 \\
\hline Líbido/Pr previa & $-.228^{*}$ & 0.02 \\
\hline Líbido/B prep & $-.215^{*}$ & 0.028 \\
\hline
\end{tabular}

Tabla 4 Sexualidad y R. Académico Fuente: Elaboración Propia

\section{Correlación de Pearson de las dimensiones Actitudes Sociales y Políticas y Percepción del Rendimiento Académico}

Se observan correlaciones de tipo negativo entre la variable tolerancia social y las variables desconfianza, comparación, inseguridad y preparación previa. Además la variable liberalismo muestra correlaciones de tipo negativo con las variables desconfianza, comparación y preparación previa. Por otro lado la variable la variable antirracismo tiene correlaciones de tipo negativo con las variables desconfianza, éxito fortuito y preparación previa. Por último, se observa que entre las variables ateísmo y preparación previa hay una correlación de tipo negativa.

Se infiere que estudiantes con actitudes de poca tolerancia social a situaciones como la unión libre, los métodos educativos liberales, la libertad de expresión o la forma de castigar a los delincuentes tienden a sentir que su rendimiento no será satisfactorio en las pruebas que realizan y sus compañeros tendrán mejores resultados que ellos además de sentir muchos nervios en los exámenes, en contraparte estudiantes de este tipo estudian con mayor tiempo de anticipación para sus exámenes.

Por otra parte se observa que los estudiantes con tendencia a actitudes liberales como la defensa de los derechos humanos, el rechazo a la esclavitud u otras formas de control muestran menor tendencia a desconfiar de su rendimiento en las pruebas a las que son sometidos en la escuela o a sentirse inferiores al resto de los compañeros, en contraparte estos estudiantes liberales y estudiantes que se identifican como ateos reconocen no estudiar con suficiente tiempo de anticipación previo un examen.
Por último es de llamar la atención que aquellos estudiantes con tendencia al racismo tienen la sensación de que sus resultados no serán los esperados y que, en caso de obtener buenos resultados será una cuestión de suerte, estos estudiantes con características racistas suelen prepararse con suficiente tiempo de anticipación para presentar sus exámenes.

\begin{tabular}{|l|c|r|}
\hline Variables correlacionadas & \multicolumn{1}{|c|}{ S } & Sign. \\
\hline Libe/Desc & $-.239^{*}$ & 0.014 \\
\hline Libe/Compa & $-.215^{*}$ & 0.028 \\
\hline Libe/Pr previa & $-.227^{*}$ & 0.02 \\
\hline Ate/Pr previa & $-.253^{* *}$ & 0.009 \\
\hline Ant/Desc & $-.298^{* *}$ & 0.002 \\
\hline Ant/Éx Fort & $-.242^{*}$ & 0.013 \\
\hline Ant/Pr previa & $-.193^{*}$ & 0.049 \\
\hline Tol soc/Desc & $-.273^{* *}$ & 0.005 \\
\hline Tol soc/Compa & $-.232^{*}$ & 0.017 \\
\hline Tol soc/Ins & $-.283^{* *}$ & 0.003 \\
\hline Tol soc/Pr previa & $-.253^{* *}$ & 0.009 \\
\hline
\end{tabular}

Tabla 5 Actitudes Políticas y R. Académico Fuente: Elaboración propia

\section{Conclusiones}

Respecto a las dimensiones ExtraversiónIntroversión y Percepción del Rendimiento Académico se concluye que conductas responsables están asociadas con la calidad y dedicación de tiempo a los estudios, sensaciones de comodidad en las evaluaciones, sentimientos positivos en torno a sí mismos y a los demás así como locus de control interno altos. Por otro lado, la sociabilidad tiene una asociación positiva con percepciones de que un buen rendimiento académico es un asunto de suerte y con el constructo de que una calificación no es un reflejo confiable del verdadero nivel de conocimiento. A su vez, la impulsividad está asociada positivamente con locus de control interno bajo y con sensaciones de inseguridad en los exámenes.

Respecto a las dimensiones EstabilidadInestabilidad y Percepción del Rendimiento Académico se concluye que la culpa está asociada de manera positiva con déficit en el tiempo y la calidad dedicada al estudio previo de los exámenes, percepciones de inseguridad, incomodidad y nerviosismo en los exámenes además de estar asociada a sentimientos de tristeza; si los resultados no son positivos y locus de control interno bajos. Niveles elevados de ansiedad se asocian a una baja calidad de estudio, sensaciones de nerviosismo, incomodidad e inseguridad en los exámenes. 
A su vez, la infelicidad está asociada a sentimientos de locus de control interno bajo, sentimientos de inferioridad, tristeza, falta de dedicación en la preparación y sensaciones de incomodidad en los exámenes. Por otra parte una alta autoestima se asocia a sensaciones de seguridad y poco nerviosismo en los exámenes así como también a la no manifestación de sentimientos de inferioridad. La hipocondría se asocia positivamente con sensaciones de incomodidad en los procesos de evaluación así como también a locus de control interno bajos.

Respecto a las dimensiones Domino-Simpatía y Percepción del Rendimiento Académico se sostiene que niveles altos de ambición están asociados positivamente con altos niveles de calidad en la preparación de los exámenes, sensaciones de comodidad en los procesos de evaluación, locus de control interno alto así como a una tendencia a no mostrar sentimientos de inferioridad.

Por otra parte, las conductas asertivas están asociadas con locus de control interno alto, sensaciones de seguridad en los exámenes y carencia de sentimientos de inferioridad. La agresividad se asocia positivamente con la tendencia a no prepararse con la suficiente calidad previa a los exámenes y una tendencia hacia la masculinidad está asociada a una percepción de que las calificaciones obtenidas no son un reflejo confiable sobre el verdadero nivel de conocimiento.

Las correlaciones derivadas de la relación entre las dimensiones Sexualidad y Percepción del Rendimiento Académico hacen concluir que niveles altos de satisfacción sexual están asociados a bajos niveles de nerviosismo, bajos niveles de inseguridad y a no tener sentimientos de inferioridad en los exámenes además de que una alta satisfacción sexual está asociada positivamente con locus de control interno alto. También se afirma que altos niveles de deseo sexual están asociados a menor calidad de estudio así como a menor dedicación de tiempo previo a los exámenes, sin embargo un alto nivel de deseo sexual se asocia a la percepción de mayor seguridad en los exámenes. Se concluye que bajos niveles de tolerancia sexual están asociados a sentimientos de inferioridad, altos niveles de desconfianza en la cantidad de los conocimientos y sensaciones de inseguridad respecto a los rendimientos esperados en los exámenes.
Respecto a las correlaciones derivadas de las dimensiones actitudes políticas-sociales y Percepción del Rendimiento Académico se sostiene que, bajos niveles de tolerancia social están asociados con altos niveles de inseguridad y desconfianza en la cantidad de conocimientos, así como a sentimientos de inferioridad; sin embargo actitudes de baja tolerancia social se asocian con mayor dedicación de tiempo en la preparación de los exámenes. A su vez altos niveles de liberalismo están asociados con mayores niveles de confianza respecto a la cantidad de conocimientos que se poseen antes de un examen y menor tendencia a manifestar sentimientos de inferioridad, pese a lo anterior una inclinación muy marcada hacia actitudes liberales se asocia con un déficit en la cantidad de tiempo dedicado a la preparación de exámenes.

De igual forma las actitudes racistas se asocian a un locus de control interno bajo y a una mayor desconfianza de que los conocimientos que se poseen sean suficientes para realizar satisfactoriamente los exámenes, en contraste, se concluye que el racismo se asocia a una mayor dedicación de tiempo previo a los exámenes.

De manera general podemos concluir que niveles altos de responsabilidad, reflexividad, autoestima y ambición están asociados positivamente con una mejor percepción de rendimiento en la vida académica mientras que niveles altos de impulsividad, ansiedad, dependencia y agresividad se asocian con una percepción negativa acerca del rendimiento académico en estudiantes universitarios de Música.

\section{Referencias}

Abbagnano, N. (1996). Diccionario de Filosofía. Mexico D. F.: Fondo de cultura economica.

Alport, G. (1966). La Personalidad. Barcelona: Herder.

Bischof, L. J. (2010). Interpretación de las Teorías de la Personalidad. México D.F.: Trillas. Brody, N., \& Ehrlichman, H. (1998). Psicología de la Personalidad. Madrid: Pearson Education.

Charles, S. C. (1997). Teorías de la Personalidad. Naucalpan de Juárez: Prentice Hall. 
Cupani, M., Garrido, S., Tavella, \& Jessica. (2013). El modelo de los cinco factores de personalidad: contrución predictiva al rendimiento académico. Revista de Psicologia, 67-86.

Cupani, M., Zalazar, J., \& Federico, M. (2014). Rasgos complejos y rendimiento académico: Contribución de los rasgos de personalidad, creencias de autoeficacia e intereses. Revista colombiana de psicología, 57-71.

Edel, R. (2003). El rendimiento académico: concepto, investigación y desarrollo. Revista electronica Iberoamericana sobre calidad, eficacia y cambio en educación.

Eysenck, H. J. (1987). Personalidad y diferencias individuales. Madrid: Pirámide.

Fueyo, E., María, M., \& Bianca, D. (2010). Personalidad eficaz y rendimiento académico: una aproximación integrada. Revista de Orientación educacional, 57-70.

G., P. (1984). Temperamento, carácter y personalidad. México D.F.: Fondo de cultura economica.

J., R. (2011). Un giro copernicano en la enseñanza universitaria: formación de competencias. Revista internacional de psicología, 21.

José, C., Lucy, R., Carmen, M., Teresa, L., \& Pedro, M. (2004). Teorías de la Personalidad. Mexico D.F.: Trillas.

Jozef, C. (1975). Evaluación de la Personalidad. Mexico D.F.: Trillas.

Kerlinger, F. (1979). Investigación del comportamiento técnicas y metodología. Mexico D.F: Interamericana.

Millon, T. (1987). Inventario Millon sobre sobre estilos de personalidad. Buenos Aires: Paidós.

Millon, T. (1996). Dissorders of Personality. Nueva York: Wiley and sons.

N., D. N. (1989). Teorías de la personalidad. Mexico D.F.: McGraw Hill.
Rios, J., García Diego, B. L., Jesús, H., \& Isela, H. (2007). Personalidad y Rendimiento Académico en estudiantes de Medicina. Congreso Virtual Internacional sobre estudios Género y Educación.

S., B. G. (1966). Teorías Psicoanalíticas de la Personalidad. Buenos Aires: Paidós.

Sampieri, H., Fernández, C., \& Pilar, B. (2008). Metodología de la Investigación. México D.F.: Ultra.

Susan, C. C. (2003). Teorías de la Personalidad. Naucalpan de Juárez: Pearson Education.

U., M. (2001). Expectativas educativas de los maestros de primaria y sus efectos en la calidad educativa.

V., S. (2010). Modelo psicobiologico de la personalidad de Eysenck:una historia proyectada hacia el futuro. Revista internacional de psicología, 1-21.

Vargas, I., Ramírez, C., J., C., A., F., \& G., H. (2011). Factores asociados al rendimiento académico en estudiantes de la facultad de medicina. Salud mental, 301-308.

Veronica, N. (2002). 